\title{
Observation of Ionization Instability of Intense Laser Pulses
}

\author{
Y. Li \\ S.P. Nikitin \\ I. Alexeev \\ H.M. Milchberg \\ T.M. Antonsen
}

This paper was prepared for submittal to the

Society of Photo-Optical Instrumentation Engineers

44th Annual Meeting of the International Symposium on

Optical Science, Engieering and Instrumentation

Denver, $\mathrm{CO}$

July 18-23, 1999

June 25, 1999

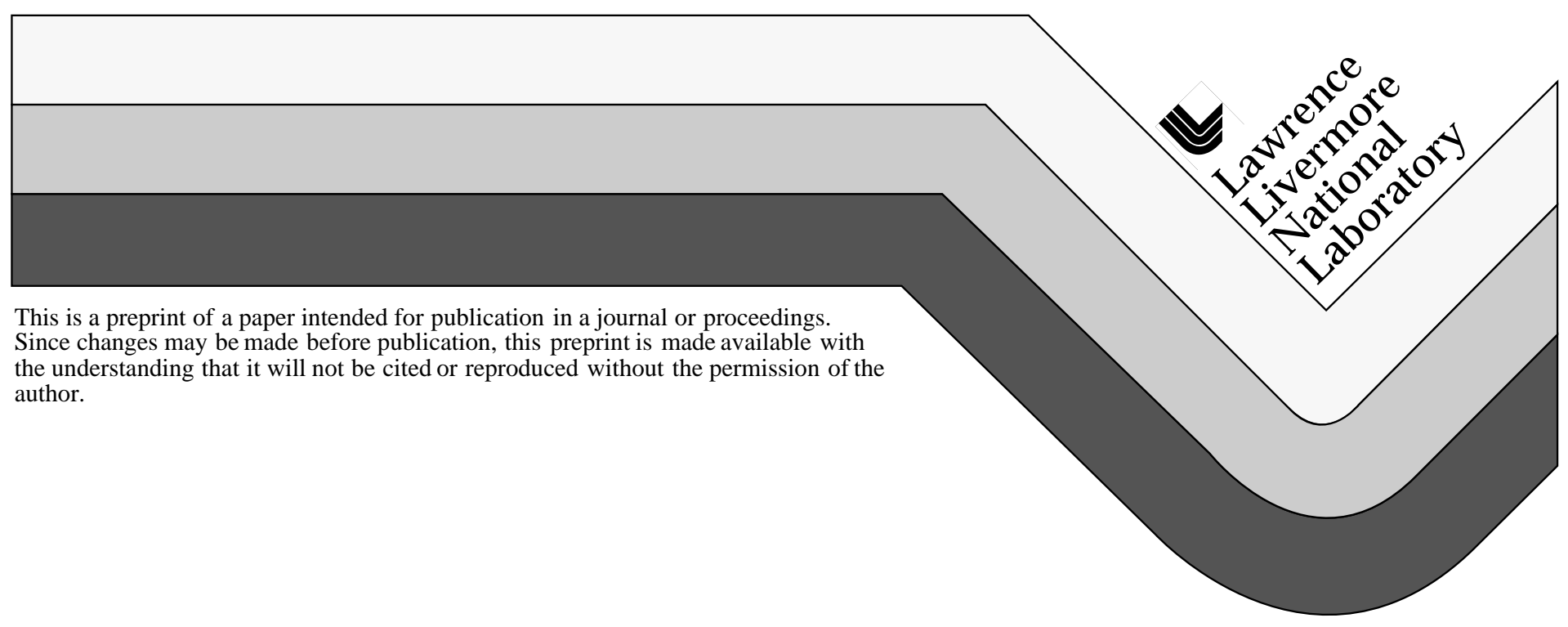




\section{DISCLAIMER}

This document was prepared as an account of work sponsored by an agency of the United States Government. Neither the United States Government nor the University of California nor any of their employees, makes any warranty, express or implied, or assumes any legal liability or responsibility for the accuracy, completeness, or usefulness of any information, apparatus, product, or process

disclosed, or represents that its use would not infringe privately owned rights. Reference herein to any specific commercial product, process, or service by trade name, trademark, manufacturer, or otherwise, does not necessarily constitute or imply its endorsement, recommendation, or favoring by the United States Government or the University of California. The views and opinions of authors expressed herein do not necessarily state or reflect those of the United States Government or the University of California, and shall not be used for advertising or product endorsement purposes. 


\title{
Observation of ionization instability of intense laser pulses
}

\author{
Yuelin $\mathrm{Li}^{*}$ \\ Institute for Laser Science and Applications, Lawrence Livermore National Laboratory \\ P. O. Box 808, L-411, Livermore, CA 94550,E-mail:ylli@LLNL.GOV \\ Sergei P. Nikitin, Ilya Alexeev, and Howard M. Milchberg \\ Institute for Physical Science and Technology, University of Maryland, College Park, MD 20742 \\ Thomas M. Antonsen \\ Institute of Plasma Research, University of Maryland, College Park, MD 20742
}

\begin{abstract}
Theoretical analysis and preliminary experiment on ionization instability of intense laser pulses in ionizing plasmas are presented. The ionization instability is due to the dependence of the ionization rate on the laser intensity and scatters the laser energy off the original propagation direction.
\end{abstract}

Keyword: ionization instability, laser propagation, laser plasmas

\section{INTRODUCTION}

Propagation of intense laser beams through gases and plasmas is of current interest not only for basic understanding of new physical phenomena, but also for its practical applications such as X-ray lasers, plasma accelerators, the fast igniter inertial confinement fusion concept, and possibly for laser triggered lightning. Intense laser propagation is normally subject to a number of instabilities such as Raman scattering, self-focusing in the relativistic regime, hose instability, and others [1]. In partially ionized plasmas, the situation is more complicated due to the nonlinear contribution from of the bound electrons and the contribution of free electrons to the dielectric response of the propagation medium [2-3].

Among these instabilities, one category that was predicted 20 years ago yet has received very little attention is the scattering of the electromagnetic wave by collective amplification of modulations of electron density due to the field dependence of the ionization rate [4-6]. Such modulations are seeded due to the nonuniformity of the transverse profile of the laser beam and generate density striations perpendicular to the oscillating laser field, which in turn scatter the laser energy and reinforce the modulation of the laser profile leading to the growth of the instability. As this can scatter the laser energy rapidly, it affects many of the applications that involve dynamic ionization. Examples are the efforts to use self focusing to overcome the ionization diffraction and propagate pulses long distances [7], and the effort to develop X-ray lasers $[8,9]$. A similar type of instability has been studied in a microwave discharge [10], but it has not yet been observed with a powerful pulse laser.

We report theoretical analysis and preliminary experimental study of the ionization instability of a high intensity ultrashort laser pulse.

\footnotetext{
* Corresponding author
} 


\section{THEORETICAL ANALYSIS}

Physically, the instability is caused by the inhomogeneous spatial profile of the laser beam, which in turn causes inhomogeneous ionization across the beam. Such ionization instantaneously modifies the local permittivity hence the local filed intensity. The modified field intensity feed back to the change in the permittivity by changing the ionization rate, and the instability grows.

Considering a laser beam propagating in a hydrogen gas, the system can be described by the wave equation of the laser electric field $E$ and the rate equation of the plasma [6],

$$
\begin{aligned}
& \left(\frac{1}{c^{2}} \frac{\partial^{2}}{\partial t^{2}}-\nabla^{2}\right) E=-k_{p}^{2} \frac{n_{e}}{n_{g}} E, \\
& \frac{\partial}{\partial t} \frac{n}{n_{g}}=v(|E|)\left(1-\frac{n_{c}}{n_{g}}\right) .
\end{aligned}
$$

Here $n_{e}$ and $n_{\mathrm{g}}$ are the densities of the electrons and the background gas, $v$ is the ionization rate, and $k_{\mathrm{p}}$ is the plasma wave vector. One can rewrite the electric field in the form of a normalized amplitude $a=e E /\left(m c^{2} k_{0}\right)$ (where $k_{0}$ is the wave vector of the laser) and express $a$ and $n_{\mathrm{e}}$ as

$$
\begin{aligned}
& a=a_{0}+\delta a_{+} e^{i k_{1}}+\delta a_{-}^{*} e^{-i k_{1} x} \\
& n=n_{0}+\delta n e^{i k_{1}}+\delta n^{*} e^{-i k_{1} x}
\end{aligned}
$$

Here $k_{\perp}$ is the wave number in transverse direction and $\delta$ denotes perturbations. Insert Eq. (3) into Eqs. (1-2), we can obtain equations describing the relatively stable "equilibrium" part and the unstable perturbation part separately. The perturbation parts are as following,

$$
\begin{aligned}
& 2 \frac{\partial}{\partial z}\left(\frac{\partial}{\partial \xi} \mp i k_{0}\right) \delta a_{ \pm}+\left(k_{p}^{2} \frac{n_{0}}{n_{g}}+k_{\perp}^{2}\right) \delta a_{ \pm}=-k_{p}^{2} \frac{\delta n}{n_{s}} a_{0}^{*}, \\
& c \frac{\partial}{\partial \xi} \frac{\delta n}{n_{g}}=\bar{v}\left(\left|a_{0}\right|\right) \frac{\delta n}{n_{g}}+c k_{1} \frac{a_{0}^{*} \delta a_{+}+\delta a_{-}}{2\left|a_{0}\right|^{2}}, \\
& k_{1}=\frac{\left|a_{0}\right|}{2 c} \frac{\partial \bar{v}}{\partial\left|a_{0}\right|}\left(1-\frac{n_{0}}{n_{g}}\right) .
\end{aligned}
$$

Here $\xi=c t-z$, and $k_{1}$ describes the coupling between the field and density perturbation. $k_{1}$ is non-zero only when ionization rate depends on field intensity and when the gas is partially ionized. Assume a slowly evolving "equilibrium" part $n_{\mathrm{e}}$ and $a_{0}$, a time and space dependence for $\delta a_{ \pm}$and $\delta n$ of the form $\exp \left[i\left(k_{\xi} \xi-k_{z} z\right)\right]=\exp (\Gamma)$, with an impulse disturbance initialized at $\xi=z=0$, one can obtain the real part of the exponential within the pulse envelope as [6]:

$$
\operatorname{Re}(\Gamma)= \pm k_{p} \sqrt{\frac{k_{1}}{k_{0}} z\left(\xi-\frac{k_{\perp}^{2}}{2 k_{0}^{2}} z\right)}-\frac{\bar{v}}{c}\left(\xi-\frac{k_{\perp}^{2}}{2 k_{0}^{2}} z\right)
$$

For $k_{\perp} \gg k_{\mathrm{p}}$, maximize $\operatorname{Re}(\Gamma)$ over $\xi$ for fixed $z$, the growth rate along the laser axis can be written as

$$
\operatorname{Re}(\Gamma)=\frac{k_{1} k_{p}^{2}}{4 k_{0}(\bar{v} / c)} z .
$$

From the above analysis, we notice several characteristics of the ionization instability. The first is its dependence on the ionization rate. Obviously, the instability grows only when the gas is ionizing, i.e., when $k_{1} \neq 0$. As a result, in gases like hydrogen and helium, the instability should be less significant than in gases like nitrogen and argon because they have less ionization stages and can be fully ionized at relatively low laser intensity. For example, He can be fully ionized at an

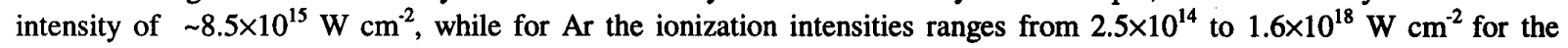
outmost 8 electrons. Secondly, high density plasmas are more subject to the instability as can be clearly seen in the Eq. (6). Finally, less obvious but equally important is the convective nature of the ionization instability. It is clear that when the 


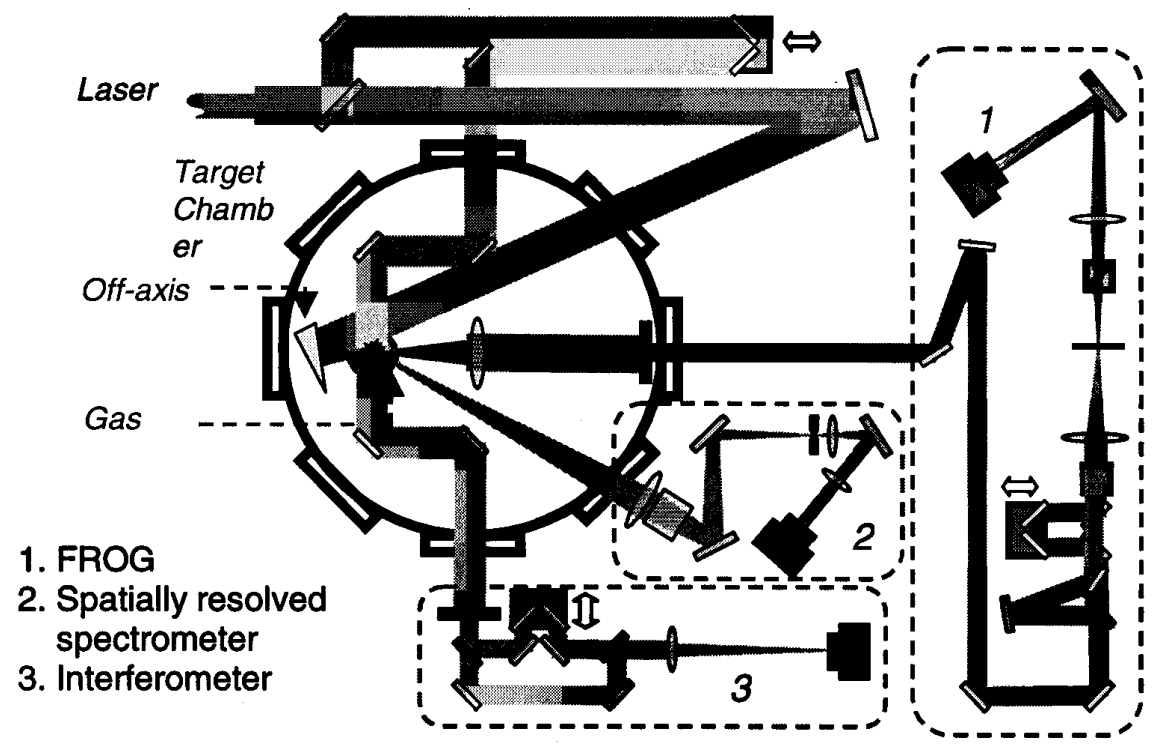

Figure 1. Schematic of the experimental setup.

transverse wave vector $k_{\perp}$ is zero no instability can grow. However, with large $k_{\perp}$, the group velocity of the of the scattered wave along the $z$ axis, i.e., $v_{\mathrm{g}} \sim c\left(1-k_{1}^{2} / k_{0}^{2}\right)^{1 / 2}$, decreases, and the will fall behind the laser pulse soon and be convected out of the unstable region. The convective nature eventually determined the angle at which the laser is scattered off the plasma.

\section{EXPERIMENTS}

\section{1 The laser facility and the experimental setup}

The experiment was performed on a home made Ti: Sapphire laser system at the Institute for Physical Science and Technology at University of Maryland. The laser consists of a $50 \mathrm{fs} \mathrm{Ti}$ : Sa oscillator, a regenerative amplifier and a two-
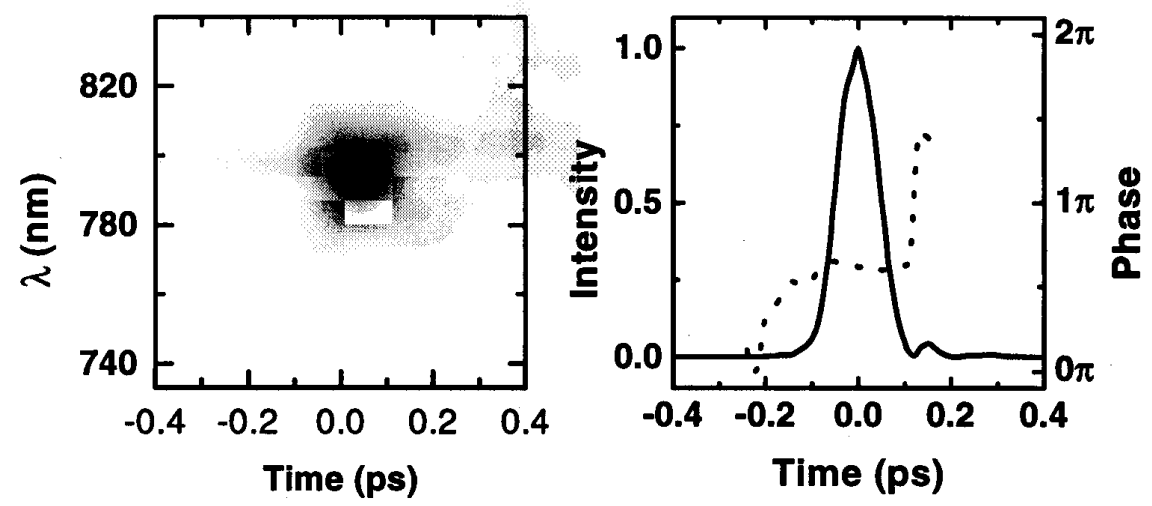

Figure 2. A typical frog trace of the pulse from the laser system. 

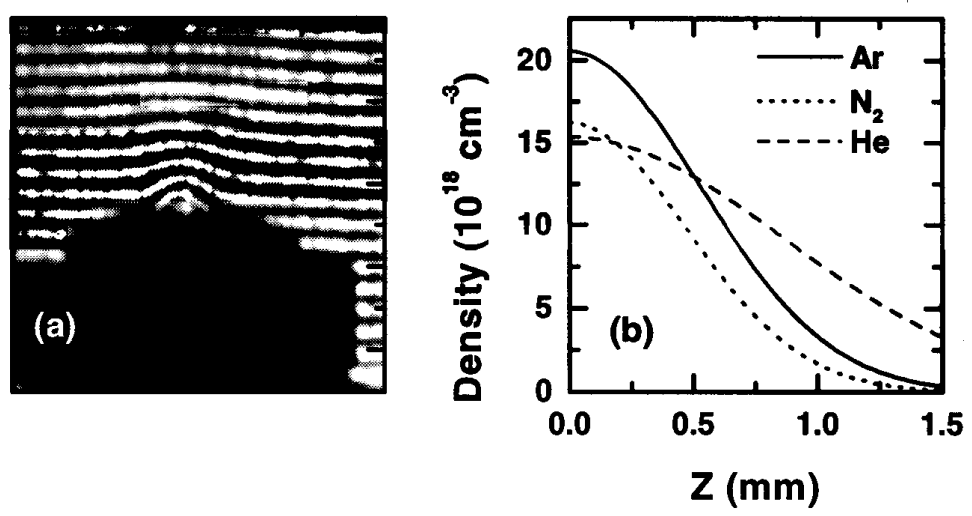

Figure 3. Measurement of the neutral density of gas jet from the nozzle. (a) A raw interferogram for Ar at 400 psi backing pressure and (b) Measured density profiles for different gases at backing pressure of $200 \mathrm{psi}$ at $0.6 \mathrm{~mm}$ above the nozzle.

stage power amplifier. The $10 \mathrm{~Hz}$ pulses from the final power amplifier are compressed to give $50 \mathrm{~mJ}, 100 \mathrm{fs}$ (FWHM) pulses at $0.79 \mu \mathrm{m}$.

To observe the ionization instability, an experimental setup as given in Fig. 1 is implemented. The laser beam is sent into the target chamber though a $\mathrm{MgF}_{2}$ window and is focused by an $\mathrm{f} / 1$ off axis parabola into a gas jet at about $0.6 \mathrm{~mm}$ above the nozzle orifice. The vacuum focal spot is elliptic with a FWHM (full width at half maximum) of $4.9 \mu \mathrm{m}$ by $2.1 \mu \mathrm{m}$ in each dimension, corresponding to a Rayleigh length of about $40 \mu \mathrm{m}$. With $50 \%$ of the energy in the focal spot, the focusing intensity is of $\sim 1.5 \times 10^{18} \mathrm{~W} \mathrm{~cm}^{-2}$ in vacuum. The input laser pulse were analyzed in situ by a frequency resolved optical gating (FROG) device [11], and reveal a near Gaussian envelope with $103 \mathrm{fs}$ FWHM and a flat phase (Fig. 2). The gas jet can be translated along the laser axis to allow the laser to sample different portion of the density profiles.

To obtain the density profile of the gas jet, an interferometer is implemented using a probe beam split from the main beam. This probe beam crosses the main beam at the interaction region and is timed through a delay line. The neutral density profile is measured and found to have a FWHM of $\sim 1.2 \mathrm{~mm}$ for all backing pressures [Fig. 3 (a) and (b)]. Because the spatial resolution of this interferometer $(-50 \mu \mathrm{m})$ is too low to obtain useful information on the plasma left after the pulse passes through the jet, in most of the experiments it was used as a shadowgraph by blocking one of the interfering
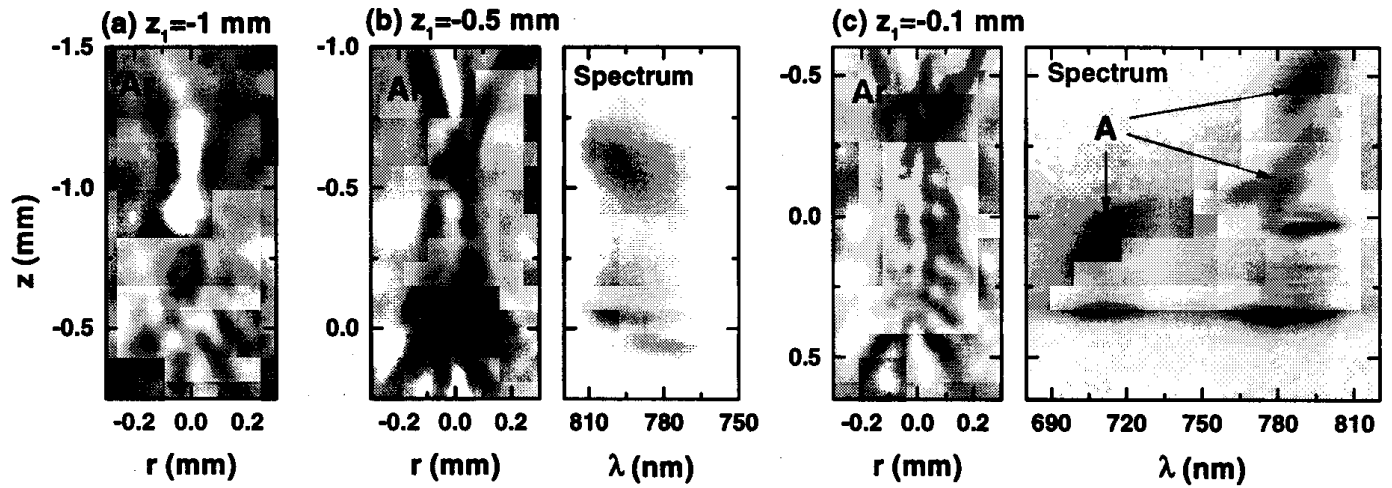

Figure. 4 The shadowgrams and the spatially resolved spectra for three cases for $\mathrm{Ar}$ at $200 \mathrm{psi}$ backing pressure, where the local atomic densities $n_{a}$ are $0.4,1.4$ and $2 \times 10^{19} \mathrm{~cm}^{-3}$, generating electron densities of $0.32,1.12,1.6 \times 10^{20} \mathrm{~cm}^{-3}$. Note the jet center is always at $z=0$. 
beams. A spectrometer is installed at an angle of $40^{\circ}$ from the laser axis. It has an acceptance angle of $\sim 10^{-2}$ ster rad and spatial resolution along the laser axis of $\sim 10 \mu \mathrm{m}$ and provides the intensity of the scattered light as a function of frequency and distance.

\subsection{Experimental results and discussion}

The spectrometer and the shadowgraph provided information both about the propagation of the laser and the plasma. A set of shadowgrams with the corresponding spatially resolved side scattered spectra for Ar at 200 psi backing pressure is given in Fig. 3. The cross calibration of the $z$ axis is believed to accurate to $-50 \mu \mathrm{m}$. Figures 4 (a), (b), and (c) show the cases when the laser, which propagates in the $z$ direction, is focused at $1,0.5$ and $0.1 \mathrm{~mm}$ before the center of the gas jet, denoted as $z_{1}=-1,-0.5$ and $-0.1 \mathrm{~mm}$. The origin $z=0$ is at the center of the gas jet. The corresponding neutral densities at the vacuum beam waist location are $n_{a} \approx 0.4,1.4$, and $2 \times 10^{19} \mathrm{~cm}^{-3}$, respectively.

In the shadowgrams, shadows reflect the phase perturbation of the probe beam due to the local increase of the electron density. Therefore, the shadowgrams, taken at about $10 \mathrm{ps}$ after the interaction, record the structure the electron density. In Fig. 4 (a), the shadowgram does not show any well defined pattern until $\sim 0.5 \mathrm{~mm}$ after the vacuum focus at which point the shadow splits into a set of structures pointing at certain angles off the laser axis. These structures are better seen in the case with $z_{1}=-0.5 \mathrm{~mm}$ in Fig. 4 (b), where the shadow suddenly reveals several 'feather' structures at $\sim 0.4 \mathrm{~mm}$ after the focus. In Fig. 4 (c), these feather structures dominate the shadow of the $\sim 0.6 \mathrm{~mm}$ propagation.

Equally interesting are the features in the spatially resolved spectra, particularly the one in Fig. 4 (c), where the spectral components can be categorized into two groups. One group is the blue shifting, weak features with smooth spatial profiles along the $z$ direction, marked $A$ in the spectrum. The second is these with sharp spatial features in the $z$ direction and higher intensity (note that a logarithmic scale is used to show the low intensity features). The differences of spectral and spatial patterns between the two suggest that they have different origins. The progressive blue shift of group A is clearly due to ionization, and can be attributed to Thomson scattering at the ionization front. The high intensity group, the positions of which have clear correlation to that of the 'feather' structures in the shadowgram, can be attributed to a coherent scattering effect of the laser. While the scattered spectrum is too weak to see in Fig. 4(a) (therefore not shown here), we observed a

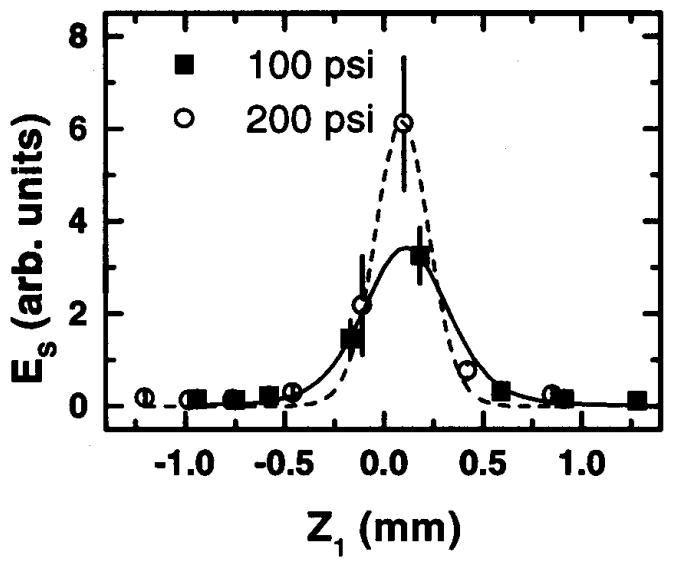

Figure 5. The measured relative total scattered energies (symbols) from the plasma as a function of the distance between vacuum focusing position and the center of the gas jet $\left(z_{1}\right)$ and the nonlinear fitting (lines) to the growth function Eq. (1) with the measured neutral density profiles for Ar at 200 and 100 psi backing pressures.

similar correlation for Fig. 4 (b).

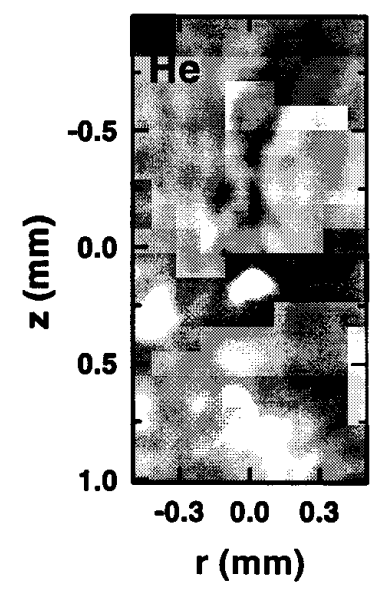

Figure 6. Shadowgram for $\mathrm{He}$ at 800 psi backing pressure with the laser focus at $z_{1}=-0.18 \mathrm{~mm}$ where $n_{\mathrm{a}} \sim 0.6 \times 10^{20} \mathrm{~cm}^{-3}$. Upon two times ionization, the electron density is $\sim 1.2 \times 10^{20}$ $\mathrm{cm}^{-3}$, roughly matching the electron density of $1.6 \times 10^{20} \mathrm{~cm}^{-3}$ in the Ar cases in Fig. 4 (b) or (c). The feather structure is much less prominent here. 

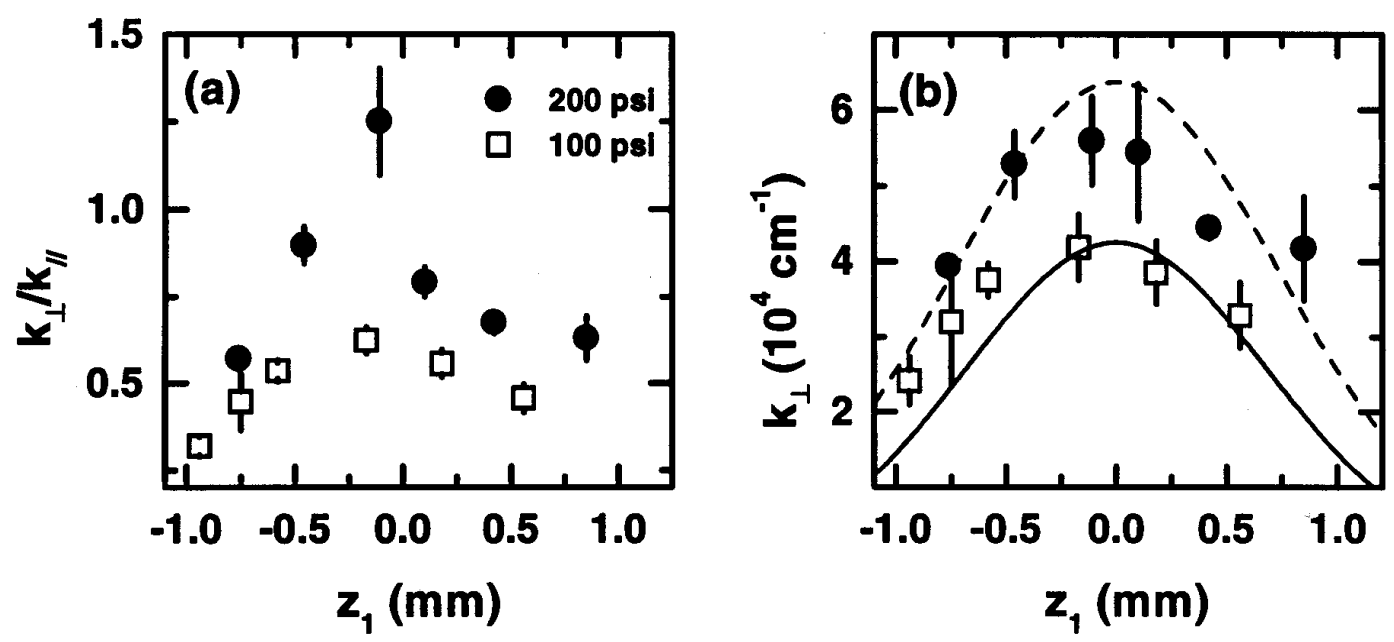

Figure 4. (a) The measured scattering angle $\left(k_{\perp} / k_{l l}\right)$ and (b) transverse wave number $k_{\perp}$ (symbols) as a function of distance between vacuum focusing position and the center of the gas jet $z_{1}$ for $\mathrm{Ar}$ at 200 and 100 psi backing pressures. The fitting of $k_{\perp}$ the to the local plasma frequency using the measured neutral density profiles is also given in (b). Dashed line: for $200 \mathrm{psi}, 2.5 k_{\mathrm{p}}$; solid line, for $100 \mathrm{psi}$, $3 k_{\mathrm{p}}$.

The correlation between the spatial position of the feathers in the shadowgram and the high intensity sharp spatial component in the spatially resolved spectra suggests the following scenario: The laser light is scattered by a certain mechanism that deflects the light off its original propagation direction and leaves ionization track along its path. The scattered light is collected by the spectrometer and the ionization tracks are captured by the shadowgram as the feathers, which approximately reveal the direction of the scattered wave.

We identify the scattering as due to the ionization instability, because it clearly displays those expected characteristics mentioned in our previous analysis.

First, with a similar initial perturbation and ionization rate, Eq. (6) suggests that the higher the plasma density (higher $k_{\mathrm{p}}$ ), the more serious the instability grows. This is can easily be verified by the difference of the shadowgrams in Fig. 4: the closer to the center of the jet the laser focuses, where the plasma density is higher, the more significant the feather structures manifest themselves.

This dependence of the growth rate on the plasma density can be more clearly illustrated by measuring the relative scattered energy. As the ionization instability grows from local spatial modes of the laser, the scattered energy is roughly proportional to the growth of the instability, or $E_{\mathrm{s}} \propto \exp \left\{z k_{1} k_{p}^{2} /\left[4 k_{0}(\bar{v} / c)\right]\right\} \propto \exp \left(\alpha n_{a}\right)$. Here $\alpha=z k_{1} /\left[4 k_{0}(\bar{v} / c)\right]$ is approximately a constant if the same gas is used and the focusing geometry is kept the same. Changing the density by translating the gas jet, this is qualitatively verified in the experiment by measuring the relative total scattered energy $E_{\mathrm{s}}$ using the spectrometer. The measured $E_{s}\left(z_{1}\right)$ is fitted to the function $\exp \left[\alpha n_{a}\left(z_{1}\right)\right]$ where $n_{a}$ is measured from the interferometer. The fitting converges surprisingly well as shown in Fig. 5, where measurement for Ar at backing pressures of 100 and 200 psi are given. The ratio of fitting factor $\alpha_{200} / \alpha_{100}$ is found to be 1.3 , close to the expected value of unity.

We also partially verified the dependence of the instability on the ionization rate using different gases, and we found the instability is most significant in Ar and least significant in He. The shadowgram for a He case with approximately equal $n_{\mathrm{e}}$ to that for Fig. 4 (b) or (c) is given in Fig. 6. The effect is strikingly clear: the feather structure is much less obvious in comparison with the Ar cases. As the wave number $k_{\mathrm{p}}$ of the plasma wave is kept the same by matching the electron 
density in both cases, the difference pinpoints the role of the ionization rate and unambiguously evidenced that the scattering is caused by the ionization instability. This is exactly the expectation from the theoretical analyzation.

The emission angle of the scattered wave is eventually determined by the convective nature of the instability which leads to competition between the local growth and the convection of the scattered wave. Longer propagation distance promotes scattering at smaller $k_{\perp}$ while shorter propagation distance favors larger $k_{\perp}$. For infinitely long propagation, $k_{\perp}$ approahces $k_{\mathrm{p}}$ as the disturbance propagates backward through the pulse at about the same rate at which the pulse is stretched due to the group velocity dispersion. For Ar at 100 and 200 psi backing pressures, the measured angle of the feathers $\left(k_{\perp} / k_{/ l}\right)$ and $k_{\perp}$ deduced from it is plotted against the position of the vacuum focus in Figs. 7 (a) and (b). We can see that the higher the plasma density, the larger the scattering angle. Fitting to the local plasma density gives $k_{\perp} \sim(2-3) k_{\mathrm{p}}$ and (34) $k_{\mathrm{p}}$ for the $200 \mathrm{psi}$ and $100 \mathrm{psi}$ backing pressure cases, respectively. This observation verifies that the instability is nonresonant.

\section{CONCLUSION}

In conclusion, we have observed plasma scattering of strong laser pulses, which can be attributed to ionization instability. To experimentally better understand this new category of instability, we plan to measure the absolute scattered energy, the transmitted energy and its signature on the transmitted pulse. Understanding of this new phenomenon is important for many applications that involve dynamic ionization of the propagation media.

\section{ACKNOWLEGEMENTS}

One of the authors (Y. Li) acknowledges the support of J. Nilsen, L. B. DaSilva, and H. Baldis. This work is supported by the National Science Foundation and the Department of Energy. Y. Li's work was also performed under the auspices of the US Department of Energy by the Lawrence Livermore National Laboratory under Contract No. W-7405-Eng-48.

\section{REFERENCES}

1. See E. Esarey et al., IEEE Trans. Plasmas Sci. 24, 252 (1996), and references therein.

2. P. Sprangle, E. Esarey, and J. Krall, Phys. Rev. E 54, 4211 (1996).

3. P. Sprangle, E. Esarey, and B. Hafizi, Phys. Rev. E 56, 5894 (1997).

4. V. B. Gil'denburg and A. V. Kim, Sov. Phys. JETP 47, 72 (1978).

5. V. B. Gil'denburg, A. G. Litvak and N. A. Zharova, Phys. Rev. Lett 78, 2968 (1997).

6. T. M. Antonsen and Z. Bian, Phys. Rev. Lett. 82, 3617 (1999).

7. S. Henz and J. Herrman, Phys. Rev. E 53, 4092 (1996).

8. B.E. Lemoff et al., Phys. Rev. Lett. 74, 1574 (1995).

9. Y. Nagata et al., Phys. Rev. Lett. 71, 3774 (1993).

10. A. L. Vikharev et al., Sov. Phys. JETP 67, 724 (1988).

11. R. Tribino and D. J. Kane, J. Opt. Soc. Am. A 10, 1102 (1993). 